\title{
Azimuthal asymmetry in HE1,X modes analyzed
}

Astorino, Antonio; Usuga Castaneda, Mario A.; Israelsen, Stine Møller; Lægsgaard, Jesper; Rottwitt, Karsten

Published in:

Frontiers in Optics 2017

Link to article, DOI:

10.1364/FIO.2017.JTu3A.87

Publication date:

2017

Document Version

Peer reviewed version

Link back to DTU Orbit

Citation (APA):

Astorino, A., Usuga Castaneda, M. A., Israelsen, S. M., Lægsgaard, J., \& Rottwitt, K. (2017). Azimuthal asymmetry in HE1,X modes analyzed. In Frontiers in Optics 2017 [JTu3A.87] Optical Society of America (OSA). https://doi.org/10.1364/FIO.2017.JTu3A.87

\section{General rights}

Copyright and moral rights for the publications made accessible in the public portal are retained by the authors and/or other copyright owners and it is a condition of accessing publications that users recognise and abide by the legal requirements associated with these rights.

- Users may download and print one copy of any publication from the public portal for the purpose of private study or research.

- You may not further distribute the material or use it for any profit-making activity or commercial gain

- You may freely distribute the URL identifying the publication in the public portal

If you believe that this document breaches copyright please contact us providing details, and we will remove access to the work immediately and investigate your claim. 


\title{
Azimuthal asymmetry in $\mathrm{HE}_{1, \mathrm{x}}$ modes analyzed
}

\author{
Antonio Astorino, Mario A. Usuga Castaneda, Stine M. Israelsen, Jesper Lægsgaard, \\ Karsten Rottwitt \\ DTU Fotonik, Ørsteds plads, building 343, 2800 Kongens Lyngby, Denmark \\ anast@fotonik.dtu.dk
}

\begin{abstract}
An analytical study of higher-order modes in step-index fibers has been conducted with the aim of justifying the circular asymmetry experimentally observed in the intensity of higher-order Bessel-like modes.
\end{abstract}

OCIS codes: $060.2310,260.2110,260.5430$.

\section{Introduction}

Fiber Bessel-like modes are attracting increasing interest due to their many applications, such as amplification [1], fourwave mixing [2], and soliton self-frequency shift [3]. In a previous work [4], the excitation of higher-order Bessel-like modes in a large-core fiber has been studied, highlighting the presence of an unexpected azimuthal asymmetry in the mode intensity. This phenomenon, denoted bowtie effect, has been ascribed to the different boundary conditions experienced by the electric field azimuthal and radial components at the core/cladding interface [4]. Nevertheless, the nature of this shape "defect," also observed in [5], has not been completely defined. Imperfections in experiments have been excluded from its possible causes by showing that also numerical results present a bowtie shape. In this work, a mathematical understanding of the bowtie effect and its connection to the experimental results are provided.

\section{Background}

When solving Maxwell's equations for an ideal step-index fiber in cylindrical coordinates $(r, \phi, z)$, it is common to find the full-vectorial solution by first writing the electric field $\mathbf{E}=\left(E_{r}, E_{\phi}, E_{z}\right)$ and magnetic field $\mathbf{H}=\left(H_{r}, H_{\phi}, H_{z}\right)$ as functions of the longitudinal components $E_{z}$ and $H_{z}$, and then use separation of variables to describe these components and obtain the characteristic equation [6]. In particular, assuming that $\Psi=\Psi(r, \phi, z, t)$ represents either $E_{z}$ or $H_{z}$, for a given mode it is possible to write $\Psi=R_{V}(r) \Phi(v \phi) \exp [i(\omega t-\beta z)]$, where $R_{V}(r)$ is the Bessel function $J_{v}$, inside the core, and the modified Bessel function $K_{v}$, in the cladding ( $v \in \mathbb{Z}$ being the function order), $\omega$ is the angular frequency of the electromagnetic wave, and $\beta$ is the mode propagation constant. Due to geometrical reasons, the function $\Phi(v \phi)$ has to meet the periodicity condition in $[0,2 \pi]$. As basis function, either $\cos (v \phi)$ or $e^{i v \phi}$ can be chosen. An arbitrary initial phase is omitted for simplicity and without losing generality.

Although sinusoidal or complex exponential functions are mathematically equivalent, the two options yield two different sets of hybrid modes, here denoted cosine-choice modes (CCMs) and exponential-choice modes (ECMs), respectively. For instance, in ECMs, a change in $\phi$ corresponds to a mere phase change in the whole field. In other words, ECMs are invariant (to within a phase factor) under rotation about the $z$-axis, and hence they all have perfectly circular intensity. Furthermore, the orthogonal field of such modes is always circularly polarized and opposite values of $v$ imply opposite rotation directions. Two identical, but counterrotating, ECMs can be added together to obtain a CCM. CCMs are always locally linearly polarized (i.e. the resulting orthogonal electric field oscillates without changing direction, although this direction is not the same everywhere) and for them the circular symmetry is not an intrinsic property. It is important to highlight that CCMs should not be confused with the conventional linearly polarized (LP) modes, for which, instead, the polarization direction is the same over the entire fiber cross section.

\section{The bowtie effect}

When $|v|=1$, the asymmetry of $\mathrm{HE}_{v, \mathrm{X}} \mathrm{CCMs}$ manifests itself as a bowtie-shaped intensity. Not even the $\mathrm{HE}_{1,1} \mathrm{CCM}$ is perfectly circular, although its angular variation is very small and difficult to perceive. The bowtie effect is more evident when $X$ is sufficiently large. Here, as an example, an ideal fiber with normalized frequency of about 45.9 is considered. The corresponding $\mathrm{HE}_{1,15}$ ECM and CCM are depicted in Figs. 1a and 1b, respectively. In these polar representations, the orientation of $\phi$ is counterclockwise and its origin is the right half of the horizontal axis (not 


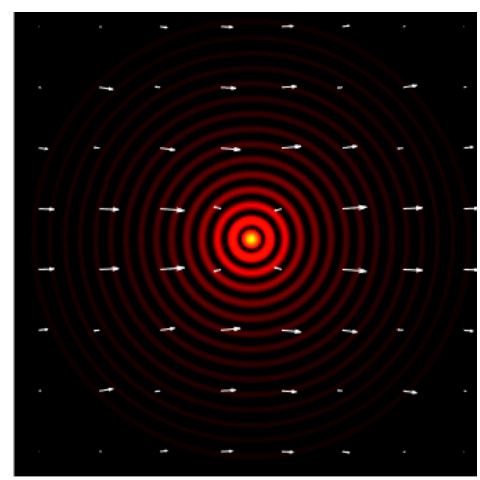

(a)

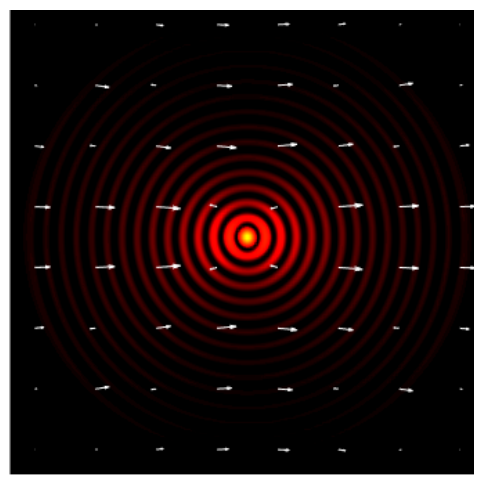

(b)

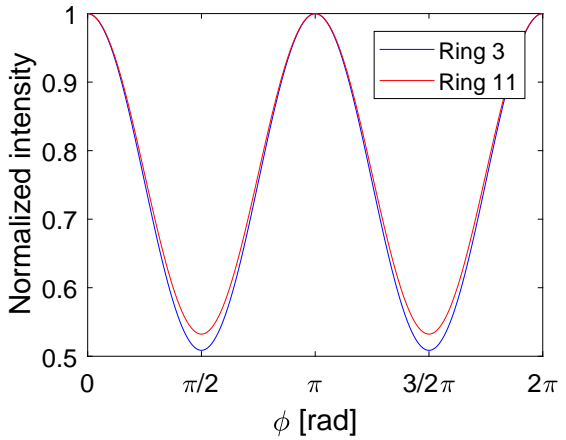

(c)

Fig. 1: Bowtie effect on $\mathrm{HE}_{1,15}$. (a) ECM and (b) CCM representations of intensity and transverse electric field vectors. (c) Intensity variation of the CCM along the crest of the $3^{\text {rd }}$ and the $11^{\text {th }}$ rings.

shown) passing through the center of the mode. The field vectors represent the real part of the transverse electric field, plotted for $\omega t-\beta z=0$ and identical in the two figures. However, as $t$ or $z$ evolves, the ECM field rotates as a whole, and hence its time-averaged intensity must be circular. The CCM, instead, maintains its orientation. Figure $1 \mathrm{c}$ represents the $\mathrm{HE}_{1,15} \mathrm{CCM}$ intensity against $\phi$ along two arbitrarily chosen rings, the $3^{\text {rd }}$ and the $11^{\text {th }}$ (counted starting from the innermost one), normalized with respect to their maximum. In Figs. 1a and 1b, it is clear that the horizontal component of the electric field is predominant, although the arrows are not perfectly aligned. This explains why, experimentally, the bowtie effect is achievable, as confirmed in [4]. In fact, when exciting a $\mathrm{HE}_{1, \mathrm{X}}$ mode with a linearly polarized source, only the component of the mode along the polarization direction of the input is excited, obtaining a field very close to that of a CCM. Consequently, the azimuthal asymmetry takes place and, in order to recover the circular shape of the "full" mode, a circularly polarized input is needed.

\section{Conclusion}

Higher-order modes do not have azimuthal symmetry if a sinusoidal function is chosen as basis for the angular dependence of the longitudinal field component. In $\mathrm{HE}_{1, \mathrm{X}}$ modes, this choice leads to an almost linear polarization, enabling the asymmetry to be reproduced also experimentally by exciting such modes with a linearly polarized input.

Acknowledgement: This project has received funding from the European Union's Horizon 2020 research and innovation programme under the Marie Sklodowska-Curie grant agreement No. 642355.

\section{References}

1. X. Peng, K. Kim, M. Mielke, T. Booth, J. W. Nicholson, J. M. Fini, X. Liu, A. DeSantolo, P. S. Westbrook, Robert S. Windeler, E. M. Monberg, F. V. DiMarcello, C. Headley, and D. J. DiGiovanni, "Higher-order mode fiber enables high energy chirped-pulse amplification," Opt. Express 21, 32411-32416 (2013)

2. J. Demas, P. Steinvurzel, B. Tai, L. Rishoj, Y. Chen, and S. Ramachandran, "Intermodal nonlinear mixing with Bessel beams in optical fiber," Optica 2, 14-17 (2015)

3. L. Rishoj, G. Prabhakar, J. Demas, and S. Ramachandran, "30 nJ, 50 fs all-fiber source at $1300 \mathrm{~nm}$ using soliton shifting in LMA HOM fiber," in Conference on Lasers and Electro-Optics, (Optical Society of America, 2016), paper STh3O.3.

4. S. M. Israelsen, L. S. Rishøj, and K. Rottwitt, "Break up of the azimuthal symmetry of higher order fiber modes." Optics Express, 22(10), 11861-11868.

5. J. U. Thomas, N. Jovanovic, R. G. Krämer, G. D. Marshall, M. J. Withford, A. Tünnermann, S. Nolte, and M. J. Steel,"Cladding mode coupling in highly localized fiber Bragg gratings II: complete vectorial analysis," Opt. Express 20, 21434-21449 (2012)

6. K. Okamoto, "Optical fibers, In Fundamentals of Optical Waveguides (Second Edition)," Academic Press, Burlington, 2006, Pages 57-67, ISBN 9780125250962 